Pacific

Journal of

Mathematics

ON AHLFORS' SCHWARZIAN DERIVATIVE AND KNOTS

MARTIN CHUAQUi

Volume $231 \quad$ No. 1

May 2007 


\title{
ON AHLFORS' SCHWARZIAN DERIVATIVE AND KNOTS
}

\author{
MARTIN CHUAQUi
}

\begin{abstract}
We extend Ahlfors' definition of the Schwarzian derivative for curves in euclidean space to include curves on arbitrary manifolds, and give applications to the classical spaces of constant curvature. We also derive in terms of the Schwarzian a sharp criterion for a closed curve in $\mathbb{R}^{3}$ to be unknotted.
\end{abstract}

\section{Introduction}

This paper is a continuation of [Chuaqui and Gevirtz 2004], in which we developed sharp bounds on the real part of Ahlfors' [1988] Schwarzian derivative for curves $C$ in $\mathbb{R}^{n}$ which imply that $C$ is simple. We begin with a geometrically simpler definition of the Schwarzian for such curves, the real part $S_{1} f$ of which coincides with that of Ahlfors. This approach has the advantage of suggesting a Schwarzian for curves in arbitrary manifolds, the results we obtain strongly suggesting that its real part, at least, is appropriately defined. After our discussion of the Schwarzian for curves in the general manifold context we focus on the particular cases of hyperbolic $n$-space $\mathbb{H}^{n}$ and the $n$-sphere $\mathbb{S}^{n}$ and derive the relationship between $S_{1} f$ as calculated with respect to the metrics on $\mathbb{H}^{n}$ and $\mathbb{S}^{n}$ on the one hand, and with respect to the euclidean metric on the underlying ball and $\mathbb{R}^{n} \cup\{\infty\}$, on the other. Using these calculations together with results of [Chuaqui and Gevirtz 2004] we obtain a very short proof of a theorem of C. Epstein [1985] to the effect a curve in $\mathbb{U}^{n}$ is necessarily simple if the absolute value of its geodesic curvature is everywhere bounded by 1 ; we also prove the theorem's spherical counterpart. Lastly, we derive a sharp bound on $S_{1} f$ which implies that the corresponding curve is unknotted.

\section{Preliminaries}

Let $f:(a, b) \rightarrow \mathbb{R}^{n}$ be a $C^{3}$ curve with $f^{\prime} \neq 0$, and let $X \cdot Y$ stand for the euclidean inner product of vectors $X, Y$ in $\mathbb{R}^{n}$. Set $|X|^{2}=X \cdot X$. As pointed out in [Chuaqui

MSC2000: primary 53A04, 53A30; secondary 53A55.

Keywords: Schwarzian derivative, simple curve, conformal metric, knot.

The author was partially supported by Fondecyt Grant \# 1030589 and by MECESUP PROJECT PUC 0103 . 
and Gevirtz 2004], the real part of Ahlfors' Schwarzian, defined by

$$
S_{1} f=\frac{f^{\prime} \cdot f^{\prime \prime \prime}}{\left|f^{\prime}\right|^{2}}-3 \frac{\left(f^{\prime} \cdot f^{\prime \prime}\right)^{2}}{\left|f^{\prime}\right|^{4}}+\frac{3}{2} \frac{\left|f^{\prime \prime}\right|^{2}}{\left|f^{\prime}\right|^{2}},
$$

can be written in terms of the velocity $v=\left|f^{\prime}\right|$ and the curvature $k$ of the trace of $f$ as

$$
S_{1} f=\left(\frac{v^{\prime}}{v}\right)^{\prime}-\frac{1}{2}\left(\frac{v^{\prime}}{v}\right)^{2}+\frac{1}{2} v^{2} k^{2},
$$

and this expression is invariant under Möbius transformations of $\mathbb{R}^{n} \cup\{\infty\}$. Our main result in that paper was:

Theorem 1. Let $p=p(x)$ be a continuous real-valued function on an open interval $I$ such that any nontrivial solution of $u^{\prime \prime}+p u=0$ has at most one zero on I. Let $f: I \rightarrow \mathbb{R}^{n}$ be a $C^{3}$ curve with $f^{\prime} \neq 0$. If $S_{1} f \leq 2 p$, then $f$ is one-to-one on $I$ and admits a spherically continuous extension to the closed interval, which is also one-to-one unless the trace of $f$ is a circle, in which case $S_{1} f \equiv 2 p$.

Although the formal expression on the right side of (1) is meaningful in the context of manifolds, its appropriateness is made apparent by the following considerations. Let $T$ denote the tangent vector along the trace of $f$, and let $\nabla$ stand for usual covariant differential operator on $M$. Then $\nabla_{T} T$ corresponds to $f^{\prime \prime}$. We regard the 2-dimensional subspace spanned by $T$ and $\nabla_{T} T$ as the complex plane $\mathbb{C}$ (the orientation being irrelevant), so that $T=a=a(t)$ and $\nabla_{T} T=b(t)$ are complex-valued functions of the parametrizing variable $t \in I$. Following the classical definition of the Schwarzian, given by

$$
\left(\frac{f^{\prime \prime}}{f^{\prime}}\right)^{\prime}-\frac{1}{2}\left(\frac{f^{\prime \prime}}{f^{\prime}}\right)^{2}
$$

we are led to consider the complex function

$$
\left(\frac{b}{a}\right)^{\prime}-\frac{1}{2}\left(\frac{b}{a}\right)^{2}
$$

as the manifold analogue of the Schwarzian. A straightforward calculation shows that the real part of the expression in (2) coincides with (1).

Let $\mathbb{G}^{n}$ denote the hyperbolic $n$-space with constant sectional curvature -1 , for which we use the standard model $\mathbb{B}^{n}=\left\{x \in \mathbb{R}^{n}:|x|<1\right\}$ with metric tensor $g_{h}=$ $4\left(1-|x|^{2}\right)^{-2} g$, where $g$ is the euclidean metric. Let $\mathbb{S}^{n}$ stand for the $n$-dimensional sphere, as modeled by $\mathbb{R}^{n} \cup\{\infty\}$ with the metric $g_{e}=4\left(1+|x|^{2}\right)^{-2} g$; here the sectional curvature is 1 . Both are special cases of a domain $\Omega \subset \mathbb{R}^{n}$ endowed with a conformal metric tensor, that is, a metric tensor of the form $\bar{g}=e^{2 \varphi(x)} g$. In this generality one can relate the Schwarzian corresponding to the resulting 
manifold $M$ with the standard euclidean Schwarzian defined on $\Omega$ itself. To do so one needs to determine how the velocity and curvature of a curve change under conformal changes of metric. Any object (velocity, curvature, covariant derivative, etc.) associated with the manifold $M$ will be distinguished from the corresponding object in the underlying $\Omega$ by a bar. Thus, let $v, k$ denote the velocity and curvature on $\Omega$ so that $\bar{v}, \bar{k}$ are their counterparts on $M$. Obviously, $\bar{v}=e^{\varphi} v$, from which routine calculations yield

$$
\begin{aligned}
& \left(\frac{\bar{v}^{\prime}}{\bar{v}}\right)^{\prime}-\frac{1}{2}\left(\frac{\bar{v}^{\prime}}{\bar{v}}\right)^{2} \\
& =\left(\frac{v^{\prime}}{v}\right)^{\prime}-\frac{1}{2}\left(\frac{v^{\prime}}{v}\right)^{2}+v^{2} \operatorname{Hess}(\varphi)(t, t)+v^{2} k(\operatorname{grad} \varphi \cdot n)-\frac{1}{2} v^{2}(\operatorname{grad} \varphi \cdot t)^{2}
\end{aligned}
$$

where $t$ and $n$ are the euclidean unitary tangent and normal vectors to the curve, $\operatorname{Hess}(\varphi)$ is the (euclidean) Hessian bilinear form and grad is the standard gradient.

In order to derive the relationship between $k$ and $\bar{k}$ one needs to know how the covariant derivative changes under conformal changes of metric. The classical formula is

$$
\bar{\nabla}_{X} Y=\nabla_{X} Y+(\operatorname{grad} \varphi \cdot X) Y+(\operatorname{grad} \varphi \cdot Y) X-(X \cdot Y) \operatorname{grad} \varphi .
$$

The curvature $\bar{k}$ is determined by the equation

$$
\bar{\nabla}_{\bar{t}} \bar{t}=\bar{k} \bar{n},
$$

where $\bar{t}=e^{-\varphi} t$ and $\bar{n}=e^{-\varphi} n$. Using (4) one obtains

$$
\bar{\nabla}_{\bar{t}} \bar{t}=e^{-2 \varphi}(k n+(\operatorname{grad} \varphi \cdot t) t-\operatorname{grad} \varphi) .
$$

After taking the euclidean norm of both sides we get

$$
\bar{k}^{2}=e^{-2 \varphi}\left(k^{2}-(\operatorname{grad} \varphi \cdot t)^{2}-2 k(\operatorname{grad} \varphi \cdot n)+|\operatorname{grad} \varphi|^{2}\right),
$$

and using (3) we have

$$
\bar{S}_{1} f=S_{1} f+v^{2} \operatorname{Hess}(\varphi)(t, t)-v^{2}(\operatorname{grad} \varphi \cdot t)^{2}+\frac{v^{2}}{2}|\operatorname{grad} \varphi|^{2} .
$$

The terms on the right-hand side depending on $\varphi$ are best expressed in terms of the Schwarzian tensor $B(\varphi)$ of the metric $\bar{g}$ with respect to $g$, as defined in [Osgood and Stowe 1992] by

$$
B(\varphi)=\operatorname{Hess}(\varphi)-d \varphi \otimes d \varphi-\frac{1}{n}\left(\Delta \varphi-|\operatorname{grad} \varphi|^{2}\right) g .
$$


Then (5) can be rewritten as

$$
\begin{aligned}
\bar{S}_{1} f & =S_{1} f+v^{2} B(\varphi)(t, t)+\frac{v^{2}}{n} \Delta \varphi+\frac{n-2}{2 n} v^{2}|\operatorname{grad} \varphi|^{2} \\
& =S_{1} f+v^{2} B(\varphi)(t, t)-\frac{v^{2}}{2} \frac{\mathrm{scal} \bar{g}}{n(n-1)} e^{2 \varphi},
\end{aligned}
$$

where scal $\bar{g}$ is the scalar curvature of the metric $\bar{g}$, that is, the sum of the sectional curvatures of any complete set orthogonal 2-planes of the tangent space at a given point. The Schwarzian tensor appears in the work of Osgood and Stowe as a suitable generalization of the classical Schwarzian derivative when studying conformal local diffeomorphisms between Riemannian manifolds, or more generally, when studying metrics on a given manifold that are conformally related. They show that conformal changes of metric with vanishing Schwarzian tensor, called Möbius changes of metric, are rare on arbitrary manifolds. On euclidean space, nevertheless, Möbius changes can be described completely and include, in particular, the hyperbolic and the spherical metric. In other words, $B(\varphi)=0$ when $e^{\varphi}$ is either $2\left(1-|x|^{2}\right)^{-1}$ or $2\left(1+|x|^{2}\right)^{-1}$.

Since scal $\bar{g}=-n(n-1)$ when $\bar{g}=g_{h}$ we obtain from (6)

$$
S_{1}^{h} f=S_{1} f+\frac{v^{2}}{2} e^{2 \varphi} .
$$

For the spherical metric we have $\operatorname{scal}(\bar{g})=n(n-1)$, hence (6) gives

$$
S_{1}^{s} f=S_{1} f-\frac{v^{2}}{2} e^{2 \varphi} .
$$

We use (7) to give a very short proof of this result:

Theorem 2 [Epstein 1985]. Let $\gamma \subset \mathbb{M}^{n}$ be a curve with geodesic curvature bounded in absolute value by 1 . Then $\gamma$ is simple.

Proof. Let $f:(-l, l) \rightarrow \gamma$ be a hyperbolic arclength parametrization. Note that the value $l=\infty$ is possible. Then $v_{h} \equiv 1$, so that $S_{1}^{h} f=k_{h}^{2} / 2$. But since $v=e^{-\varphi}=$ $\left(1-|x|^{2}\right) / 2$ it follows from (7) that

$$
S_{1} f=\frac{k_{h}^{2}-1}{2} \leq 0 .
$$

By appealing to Theorem 1 with the choice $p(x) \equiv 0$, we conclude that $\gamma$ is simple.

In the same vein, we can use (8) to derive the corresponding simplicity criterion for curves on $\mathbb{S}^{n}$. 
Theorem 3. Let $\gamma \subset \mathbb{S}^{n}$ be a curve of length $l \leq 2 \pi$ and geodesic curvature $k_{s}$ satisfying

$$
k_{s}^{2} \leq \frac{4 \pi^{2}-l^{2}}{l^{2}} .
$$

Then $\gamma$ is simple except when it is a circle of constant curvature $\sqrt{4 \pi^{2}-l^{2}} / l$.

Proof. We proceed as before and consider $f:[0, l] \rightarrow \gamma$, a spherical arclength parametrization. Then $S_{1}^{s} f=k_{s}^{2} / 2$ and $v_{s}=\left(1+|x|^{2}\right) / 2$, so (8) gives

$$
S_{1} f=\frac{1+k_{s}^{2}}{2} \leq \frac{2 \pi^{2}}{l^{2}} .
$$

This time we apply Theorem 1 with $p(x) \equiv \pi^{2} / l^{2}$ to conclude that $f((0, l))$ is simple. The extended curve $f([0, l])$ remains simple unless it is a circle, of constant curvature $\sqrt{4 \pi^{2}-l^{2}} / l$.

\section{Knots}

Theorem 4. Let $f:[-1,1) \rightarrow \mathbb{R}^{3}$ parametrize a simple closed curve in $\mathbb{R}^{3}$. If the periodic continuation of $f$ is $C^{3}$ and $S_{1} f(t) \leq 2 \pi^{2}$ for all $t \in(-1,1)$, then $f([-1,1))$ is unknotted.

Proof. The idea is to show that, if knotted, the curve $\Gamma=f([-1,1])$ can be laid out to form a planar, closed, nonsimple curve for which the real part of the Schwarzian has not increased. The process used to do this is based on the following ideas, developed by Brickell and Hsiung [1974] in the course of their proof of the FaryMilnor theorem.

For $p \in \mathbb{R}^{3}$ we define the shell $C_{p}$ of $\Gamma$ with vertex $p$ to be the developable surface made up of all segments $[p, q]$ with $q \neq p$ on $\Gamma$. The indicatrix of $C_{p}$, denoted by $I_{p}$, is the curve on $\mathbb{S}^{2}=\left\{u \in \mathbb{R}^{3}:|u|=1\right\}$ traced by the vectors $(q-p) /|q-p|$; its length $l\left(I_{p}\right)$ is called the total angle of $I_{p}$. A key fact established by Brickell and Hsiung is that $\Gamma$ is unknotted if $l\left(I_{p}\right)<3 \pi$ for all $p \in \Gamma$. The proof of this uses Crofton's formula

$$
\int n(G) d G=4 l\left(I_{p}\right)
$$

giving the length of $I_{p}$ in terms of the number $n(G)$ of intersection points of $I_{p}$ with great circles $G \subset \mathbb{S}^{2}$. The integral is performed over $\mathbb{S}^{2}$, after identifying a point on the sphere with the normal direction of a plane containing a great circle. The authors show that $n(G) \geq 1$ for all $G$ and that $\{G: n(G)=2\}$ has measure zero [Brickell and Hsiung 1974, Lemma 8, p. 188]. Since the measure of the entire set of great circles is $4 \pi$, if $l\left(I_{p}\right)<3 \pi$ then $\{G: n(G)=1\}$ must have positive measure. Hence there exists at least one great circle $G$ with $n(G)=1$, which means 
there exists one plane through the point $p$ intersecting $\Gamma$ at exactly one other point $q \neq p$. Such a plane is called transversal to $\Gamma$. The curve $\Gamma$ has the transversal property if for any $p \in \Gamma$ there exists a plane through $p$ transversal to $\Gamma$.

Theorem [Brickell and Hsiung 1974, Lemma 6, p. 191]. Let C be a closed smooth curve embedded in hyperbolic or euclidean space of dimension three. If $C$ has the transversal property then $C$ is a trivial knot.

We conclude from this discussion that if $\Gamma$ is a knot there is a point $p \in \Gamma$ for which $l\left(I_{p}\right) \geq 3 \pi$. The two cases $l\left(I_{p}\right)>3 \pi$ and $l\left(I_{p}\right)=3 \pi$ require a slightly different analysis. Suppose first that $l\left(I_{p}\right)>3 \pi$. As we move $p$ to a point $p^{\prime}$ slightly away from $\Gamma$, the number $l\left(I_{p^{\prime}}\right)$ varies continuously, except for a jump increment by $\pi$. Hence there exists $p^{\prime} \notin \Gamma$ for which $l\left(I_{p^{\prime}}\right)>4 \pi$. On the other hand, since $l\left(I_{r}\right)$ is a continuous function of $r \in \mathbb{R}^{3} \backslash \Gamma$ and since $l\left(I_{r}\right) \rightarrow 0$ as $|r| \rightarrow \infty$, we can find $p_{0} \notin \Gamma$ such that $l\left(I_{p_{0}}\right)=4 \pi$. We now lay out the shell $C_{p_{0}}$ isometrically onto the plane so that $\Gamma$ traces out a closed curve $\gamma$ that is not simple. To do this, let $\Gamma=\Gamma(s)$ be an arclength parametrization, $0 \leq s \leq L$, and set $r(s)=\left|\Gamma(s)-p_{0}\right|$. We lay out $\Gamma$ onto the plane curve $\gamma$ given by $z=z(s)=r(s) e^{i \theta(s)}$, where the function $\theta$ is chosen so that $\left|z^{\prime}(s)\right|=1$, or equivalently

$$
\left|r^{\prime}(s)+\operatorname{ir}(s) \theta^{\prime}(s)\right|=1 .
$$

The function

$$
\theta(s)=\int_{0}^{s} \frac{\sqrt{1-\left(r^{\prime}(t)\right)^{2}}}{r(t)} d t
$$

has this property. The point $p_{0}$ corresponds to $z=0 \notin \gamma$, and the polar angle $\theta=\theta(s)$ increases at the same rate as the spatial angle of the rays $\left[p_{0}, \Gamma(s)\right]$ at the vertex $p_{0}$. Because $l\left(I_{p_{0}}\right)=4 \pi$ it follows that $\gamma$ is a closed curve with winding number 2 with respect to the origin.

If, on the other hand, $l\left(I_{p}\right)=3 \pi$, we let $p_{0}=p$ and lay out $\Gamma$ as before. We may assume that $p_{0}=\Gamma(0)$. Since the point $p_{0}$ belongs to $\Gamma$, the curve $\gamma$ obtained is closed because $r(s) \rightarrow 0$ as $s \rightarrow 0^{+}$and as $s \rightarrow L^{-}$. Also, because $\Gamma$ possesses a tangent at $p_{0}$, it is easy to see that the integrand in the equation for $\theta(s)$ above behaves like $h(s) / \sqrt{s(L-s)}$, where $h$ is continuous on $[0, L]$. In other words, $\gamma(s)=z(s)$ is a planar curve passing through $z=0$ with the property that $\theta(s)=\arg \{z(s)\}$ is increasing and has total variation of $3 \pi$. A variant of the argument principle allowing for zeros on the curve (see [Nehari 1952, p. 131], for instance) implies that $\gamma$ cannot be simple: the point $0 \in \gamma$ contributes $\pi$ to the total variation of argument and therefore $\gamma$ must in addition wind around the origin once.

In either case, let $g:[-1,1) \rightarrow \mathbb{R}^{2}$ be the induced parametrization of $\gamma$ defined on the original interval of definition of $f$. We claim that $S_{1} g \leq S_{1} f$. First, $v_{g}=$ 
$\left|g^{\prime}\right|=\left|f^{\prime}\right|=v_{f}$ because the laying-out process preserves arclength. Secondly, the term involving the curvature does not increase because the curvature of $\gamma$ is equal to the curvature of $\Gamma$ relative to the surface $C_{p_{0}}$, that is, equal to the length of the projection of the curvature vector of $\Gamma$ in $\mathbb{R}^{3}$ onto the tangent plane to the shell. We see from (1) that $S_{1} g \leq S_{1} f$.

Since $\gamma$ is not simple, it can be subdivided into closed curves $\gamma_{1}, \gamma_{2}$ that are differentiable except at the point where $\gamma$ self-intersects. Because $g$ is periodic, one can find intervals $[a, b]$ and $[c, d]$ of total length 2 such that

(i) $g_{1}=\left.g\right|_{[a, b]}:[a, b] \rightarrow \gamma_{1}$ and $g_{2}=\left.g\right|_{[c, d]}:[c, d] \rightarrow \gamma_{2}$, and

(ii) the parametrizations $g_{1}, g_{2}$ are $C^{3}$ on the open subintervals.

This sketch represents the case when $p_{0} \notin \Gamma$ together with the corresponding nonsimple curve $g$ :
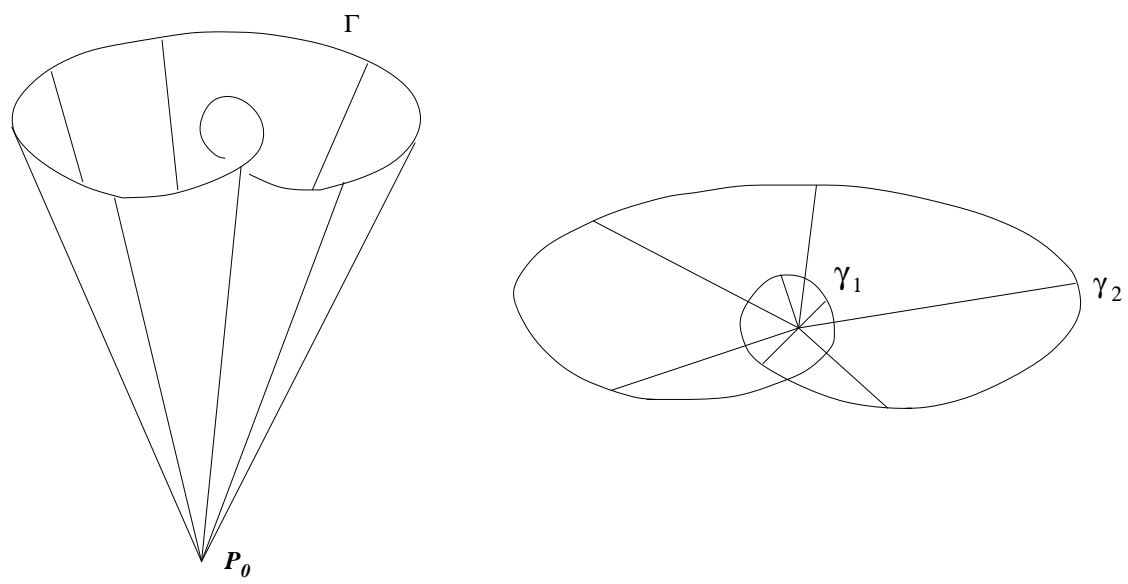

We will show that both $\gamma_{1}$ and $\gamma_{2}$ are circles and that and each subinterval $[a, b]$, $[c, d]$ has length 1 . In effect, it follows from Theorem 1 that the optimal $C$ constant for a univalence criterion $S_{1} h \leq C$ on an open interval of length $d$ is $C=2 \pi^{2} / d$, and that the extended curve can be closed only if it is a circle and $S_{1} h \equiv 2 \pi^{2} / d$. Because $S_{1} g_{1}, S_{1} g_{2}$ are bounded above by $2 \pi^{2}$ on the open intervals and the curves $\gamma_{1}$ and $\gamma_{2}$ are closed, we conclude that the length of each subinterval $[a, b],[c, d]$ cannot be less than 1 . Because the total length is 2 , each subinterval must have length 1 , and since $\gamma_{1}$ and $\gamma_{2}$ are closed, they must be circles with $S_{1} g_{1}=S_{1} g_{2} \equiv 2 \pi^{2}$. Hence $S_{1} g \equiv 2 \pi^{2}$, which can only happen if $S_{1} f \equiv 2 \pi^{2}$ and the curvature of $\gamma$ remains the same as that of $\Gamma$. Hence $\Gamma$ is an asymptotic curve, that is, the normal curvature vanishes at each point of $\Gamma$. Because the segments $\left[p_{0}, q\right]$ on the shell $C_{p_{0}}$ are lines of curvature with corresponding principal curvature equal to zero, it follows that either $\Gamma$ lies entirely on one such segment or else the shell is planar. 
In the first case, $\Gamma$ could not be closed, and in the second, it could not be knotted. This contradiction proves the theorem.

\section{Example}

In this final section we will show with that the assumption in Theorem 4 that the periodic continuation of $f$ be smooth is essential. We will construct a closed curve $f:[-1,1] \rightarrow \mathbb{R}^{3}$ with $S_{1} f \leq 2 \pi^{2}$ on $(-1,1)$, whose image is a knot that is not of class $C^{3}$ at $f(1)=f(-1)$. The function $f$ will be a Möbius transformation of the following curve $g$.

Let $g:(-1,1) \rightarrow \mathbb{C}$. We write

$$
S_{1} g=\left(\frac{v^{\prime}}{v}\right)^{\prime}-\frac{1}{2}\left(\frac{v^{\prime}}{v}\right)^{2}+\frac{1}{2} k^{2} v^{2}=2 q+\frac{1}{2} k^{2} v^{2} .
$$

We will make $S_{1} g \leq 2 \pi^{2}$ everywhere on the open interval, but with different weights for the terms $2 q=\left(v^{\prime} / v\right)^{\prime}-(1 / 2)\left(v^{\prime} / v\right)^{2}$ and $k^{2} v^{2} / 2$. Intuitively, the term $q$ determines how fast one traverses the curve, while the second term determines the shape.

Let $\delta>0$ be small. On $\left[-\frac{1}{2}+\delta, \frac{1}{2}-\delta\right]$ the curve $g$ will have $q \equiv 0, v \equiv 1$ and $k \equiv 2 \pi$. In other words, on this interval $g$ describes almost a complete circle. We define $g$ on $\left(\frac{1}{2}-\delta, 1\right)=\left(\frac{1}{2}-\delta, \frac{1}{2}+\delta\right] \cup\left(\frac{1}{2}+\delta, 1\right)$, and on $\left(-1,-\frac{1}{2}+\delta\right)$ in a symmetric way. On $\left(\frac{1}{2}-\delta, \frac{1}{2}+\delta\right.$ ] we increase the value of $q$ smoothly; this produces an increment in $v$, which forces us to decrease the value of $k$. We will do this in a way that

$$
\int_{\frac{1}{2}-\delta}^{\frac{1}{2}+\delta} k v d x=\int k d s=2 \pi \delta .
$$

Because of the symmetry on $\left[-\frac{1}{2}-\delta,-\frac{1}{2}+\delta\right)$ we will have

$$
\int_{-\frac{1}{2}-\delta}^{\frac{1}{2}+\delta} k v d x=\left(\int_{-\frac{1}{2}-\delta}^{-\frac{1}{2}+\delta}+\int_{-\frac{1}{2}+\delta}^{\frac{1}{2}-\delta}+\int_{\frac{1}{2}-\delta}^{\frac{1}{2}+\delta}\right) k v d x=2 \pi .
$$

On the remaining interval $\left(\frac{1}{2}+\delta, 1\right)$ we will decrease $k$ sharply to 0 , shifting all the weight to $q \equiv \pi^{2}$. Therefore, $g$ will map this interval to a straight line. We will show that this can done in a way that the value of $v^{\prime} / v$ at $x=\frac{1}{2}+\delta$ is large enough to allow the parametrization of a straight line with $S_{1} g=2 \pi^{2}$ on an interval of length $\frac{1}{2}-\delta$ to reach the point at infinity.

The details are as follows: 
(I) The interval $\left(\frac{1}{2}-\delta, \frac{1}{2}+\delta\right]$ : We see from (9) that $k v=\sqrt{4 \pi^{2}-4 q}=2 \pi \sqrt{1-h}$, where $h=q / \pi^{2}$. From (10) we seek $h=h(x) \in[0,1]$ such that

$$
\int_{\frac{1}{2}-\delta}^{\frac{1}{2}+\delta} \sqrt{1-h} d x=\delta
$$

If we shift the interval in question to $(0,2 \delta]$, we can choose $h$, for example, so that

$$
\sqrt{1-h(x)}=1-\frac{x}{2 \delta}
$$

that is,

$$
h(x)=\frac{x}{\delta}-\left(\frac{x}{2 \delta}\right)^{2}
$$

(This choice requires only to be smoothed out at the endpoints of the interval.) With this,

$$
\int_{0}^{2 \delta} \sqrt{1-h} d x=2 \delta-\frac{1}{2 \delta} \frac{(2 \delta)^{2}}{2}=\delta
$$

Observe that

$$
\int_{0}^{2 \delta} h d x=\int_{0}^{2 \delta}\left(\frac{x}{\delta}-\left(\frac{x}{2 \delta}\right)^{2}\right) d x=\frac{(2 \delta)^{2}}{2 \delta}-\frac{(2 \delta)^{3}}{3(2 \delta)^{2}}=\frac{4 \delta}{3},
$$

a fact that will be important ahead.

(II) The term $v^{\prime} / v$ : Let $y=v^{\prime} / v$. Then

$$
y^{\prime}=2 q+\frac{1}{2} y^{2}=2 \pi^{2} h+\frac{1}{2} y^{2} .
$$

For convenience, once more we replace the interval $\left(\frac{1}{2}-\delta, \frac{1}{2}+\delta\right]$ by $(0,2 \delta]$. The initial condition for (12) is $y(0)=0$. We want to know whether $y(2 \delta)$ (which corresponds to the original value of $v^{\prime} / v$ at $\frac{1}{2}+\delta$ ) is sufficiently large so that the parametrization of a straight line with velocity $v=e^{\int y d x}$ reaches the point at infinity before time $\frac{1}{2}-\delta$.

The parametrization of a straight line with Schwarzian identically equal to $2 \pi^{2}$ reaches the point at infinity in time exactly $\frac{1}{2}$ if its initial velocity has $v^{\prime}=0$. To verify this we consider the differential equation

$$
w^{\prime}=2 \pi^{2}+\frac{1}{2} w^{2}, \quad w(0)=0,
$$

which has the solution $w(x)=2 \pi \tan (\pi x)$. The corresponding parametrization of the straight is then given by $\frac{1}{\pi} \tan (\pi x)$, which indeed becomes infinite at $x=\frac{1}{2}$. Now we need to verify that the solution $y$ of (12) has

$$
y(2 \delta)>w(\delta) .
$$


By integrating (12) we see from (11) that

$$
y(2 \delta)>2 \pi^{2} \int_{0}^{2 \delta} h d x=\frac{8 \pi^{2} \delta}{3},
$$

while

$$
w(\delta)=2 \pi^{2} \delta+O\left(\delta^{3}\right),
$$

so that (13) will hold if $\delta$ is small enough. Thus $g$ reaches the point at infinity symmetrically at $1-\epsilon$ and $-1+\epsilon$, for some $\epsilon=O(\delta)$. In order to rectify the fact that $g$ is defined only on $(-1+\epsilon, 1-\epsilon)$, we consider the scaled parametrization $g((1-\epsilon) x)$ defined on $(-1,1)$, the Schwarzian of which is equal to $(1-\epsilon)^{2} S_{1} g<$ $2 \pi^{2}$. We keep the notation $g$ for the scaled curve; its trace together with the knot to be produced are shown in the following figure.
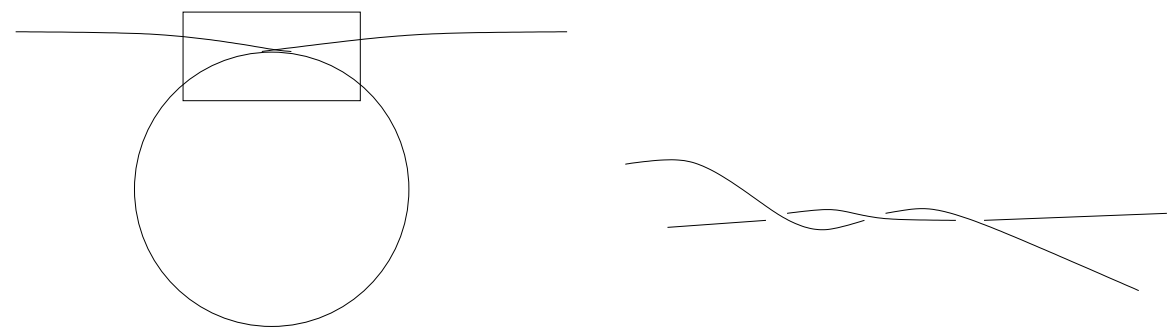

In the final step we produce a knot on $g$ with a very small cost in $S_{1} g$. The knot can be accomplished by replacing a small portion of one of the arcs at the point of self-intersection of $g$ by a very thin tubular neighborhood, along which the new arc of $g$ will go around once. Although this procedure introduces torsion, $S_{1} g$ does not depend on it. It is easy to see that both the modified curvature and velocity remain arbitrarily close to their original values as long as the tubular neighborhood is thin enough. To finish the construction, we consider some Möbius transformation $T$ for which $f=T(g)$ lies in the finite plane.

\section{Acknowledgements}

We thank Julian Gevirtz for many conversations regarding this paper and the proof of Theorem 4 in particular, as well as for bringing to our attention the work of Brickell and Hsiung.

\section{References}

[Ahlfors 1988] L. V. Ahlfors, "Cross-ratios and Schwarzian derivatives in $\mathbf{R}^{n}$ ", pp. 1-15 in Complex analysis: Articles dedicated to Albert Pfluger, edited by J. Hersch and A. Huber, Birkhäuser, Basel, 1988. MR 90a:30055 Zbl 0675.30021 
[Brickell and Hsiung 1974] F. Brickell and C. C. Hsiung, "The total absolute curvature of closed curves in Riemannian manifolds", J. Differential Geometry 9 (1974), 177-193. MR 49 \#3795 Zbl 0277.53004

[Chuaqui and Gevirtz 2004] M. Chuaqui and J. Gevirtz, "Simple curves in $\mathbb{R}^{n}$ and Ahlfors' Schwarzian derivative", Proc. Amer. Math. Soc. 132:1 (2004), 223-230. MR 2005f:53001 Zbl 1045.53003

[Epstein 1985] C. L. Epstein, "Envelopes of horospheres and Weingarten surfaces in hyperbolic 3-space”, preprint, Princeton Univ., 1985.

[Nehari 1952] Z. Nehari, Conformal mapping, McGraw-Hill, New York, 1952. Reprinted Dover, New York, 1975. MR 51 \#13206 Zbl 0048.31503

[Osgood and Stowe 1992] B. Osgood and D. Stowe, "The Schwarzian derivative and conformal mapping of Riemannian manifolds", Duke Math. J. 67:1 (1992), 57-99. MR 93j:53062 Zbl 0766. 53034

Received December 26, 2005. Revised September 5, 2006.

\author{
MARTIN ChUAQUi \\ FACUlTad de Matemáticas \\ P. Universidad Católica de Chile \\ CASILla 306 \\ SANTIAGO 22 \\ CHILE \\ mchuaqui@mat.puc.cl \\ www.mat.puc.cl
}

